\title{
Epstein-Barr Viral Load in Cerebrospinal Fluid as a Diagnostic Marker of Central Nervous System Involvement of AIDS-related Lymphoma
}

\author{
Kunio Yanagisawa ${ }^{1}$, Junko Tanuma ${ }^{2}$, Shotaro Hagiwara ${ }^{3}$, Hiroyuki Gatanaga ${ }^{2}$, \\ Yoshimi Kikuchi ${ }^{2}$ and Shinichi Oka ${ }^{2}$
}

\begin{abstract}
Objective AIDS-related lymphoma (ARL) often involves the central nervous system (CNS). Although the diagnostic value of Epstein-Barr virus (EBV)-DNA in cerebrospinal fluid (CSF) in detecting HIV-positive primary CNS lymphoma (PCNSL) has been established, its usefulness for identifying CNS involvement of systemic ARL remains elusive. In this study, we evaluated the utility of the EBV-DNA load in CSF in identifying CNS involvement in patients with systemic ARL.

Methods We retrospectively reviewed the clinical and pathological data of consecutive ARL patients managed at our clinic between January 1998 and June 2012. Sixty-two patients with ARL, including eight PCNSL patients and 52 systemic ARL patients, and 63 controls underwent CSF EBV-DNA load evaluations before receiving chemotherapy. ARL-related CNS involvement was defined as any lesion diagnosed histologically or radiologically as a lymphoma in the brain, meninges, spine, cranial nerves or oculus.

Results A cut off value of 200 copies/mL predicted the presence of CNS lesions with a sensitivity of $70 \%$ and a specificity of $85 \%$ in both the PCNSL and systemic ARL patients, while a sensitivity of $75 \%$ and a specificity of $93 \%$ were obtained for systemic ARL. A cut off value of 2,000 (3.30 log) copies/mL provided the best specificity $(100 \%)$, with a sensitivity of $50 \%$.

Conclusion Our results support the clinical utility of evaluating the quantitative EBV-DNA load in the CSF for the diagnosis of CNS involvement of systemic ARL as well as PCNSL.
\end{abstract}

Key words: AIDS-related lymphoma, Epstein-Barr virus

(Intern Med 52: 955-959, 2013)

(DOI: 10.2169/internalmedicine.52.9088)

\section{Introduction}

Although the incidence of AIDS-related lymphoma (ARL) has decreased following the advent of highly active antiretroviral therapy (HAART), the morbidity and mortality associated with this complication remain significant due to the aggressive clinical course and high frequency of extra nodal localization especially in the central nervous system (CNS) (1-3). Since the majority of patients with ARLs are diagnosed at the advanced stage of HIV infection, making the differential diagnosis of CNS lesions from other oppor- tunistic diseases is crucial for the management of ARL.

Epstein-Barr virus (EBV) can cause various lymphoproliferative disorders in immunocompromised patients and the detection of EBV-DNA in the cerebrospinal fluid (CSF) is a well-established diagnostic tool for identifying primary CNS lymphoma (PCNSL) in HIV-infected individuals (3-10). However, the diagnostic value of detecting EBV-DNA in CNS involvement of systemic ARL remains to be elucidated. In this study, we retrospectively evaluated the value of EBV-DNA in the diagnosis of CNS lesions of ARL, both PCNSL and systemic ARL.

\footnotetext{
${ }^{1}$ Department of Medicine and Clinical Science, Graduate School of Medicine, Gunma University, Japan, ${ }^{2}$ AIDS Clinical Center, National Center for Global Health and Medicine, Japan and ${ }^{3}$ Department of Hematology, National Center for Global Health and Medicine, Japan Received for publication October 9, 2012; Accepted for publication January 16, 2013

Correspondence to Dr. Junko Tanuma, jtanuma@acc.ncgm.go.jp
} 
Table. Characteristics of the Participating Patients

\begin{tabular}{|c|c|c|c|c|c|}
\hline & \multirow[b]{2}{*}{$\begin{array}{l}\text { PCNSL } \\
(n=8)\end{array}$} & \multicolumn{2}{|c|}{ Systemic ARL } & \multirow[b]{2}{*}{$\begin{array}{l}\text { non-ARL control } \\
\text { subjects }(n=63)\end{array}$} & \multirow[b]{2}{*}{$\mathrm{p}$ value } \\
\hline & & $\begin{array}{l}\text { CNS involvement }(+) \\
(\mathrm{n}=12)\end{array}$ & $\begin{array}{c}\text { CNS involvement (-) } \\
(\mathrm{n}=42)\end{array}$ & & \\
\hline Male sex, $\mathrm{n}$ & 8 & 10 & 41 & 60 & 0.981 \\
\hline Age, median years (range) & $38(28-53)$ & $52(27-67)$ & $37(25-63)$ & $38(22-70)$ & 0.160 \\
\hline \multicolumn{6}{|l|}{ Histology } \\
\hline DLBCL & 3 & 6 & 16 & - & \\
\hline Burkitt & 0 & 4 & 16 & - & \\
\hline Others & 2 & 1 & 10 & - & \\
\hline Not specified & 3 & 1 & 0 & - & \\
\hline EBER-positive, \% (n/total n) & $40(2 / 5)$ & $40(4 / 10)$ & $58.3(21 / 36)$ & - & 0.999 \\
\hline CD4 count, median cells $/ \mathrm{mm}^{3}$ (range) & $18(2-79)$ & $83(3-652)$ & $117(3-824)$ & $57(1-450)$ & 0.006 \\
\hline Plasma HIV viral load, median log copies/mL (range) & $5.8(4.5-6.0)$ & $4.7(1.6-7.1)$ & $4.7(1.6-7.5)$ & $4.6(1.7-6.3)$ & 0.081 \\
\hline Plasma EBV-DNA-positive, \% (n/total n) & $66.7(4 / 6)$ & $63.6(7 / 11)$ & $58.3(21 / 36)$ & NA & 0.999 \\
\hline CSF EBV-DNA-positive, \% (n/total n) & $62.5(5 / 8)$ & $75.0(9 / 12)$ & $7.1(3 / 42)$ & $20.6(13 / 63)$ & 0.035 \\
\hline
\end{tabular}

PCNSL: primary CNS lymphoma, ARL: AIDS-related lymphoma, DLBCL: diffuse large B-cell lymphoma, EBER: EBV-encoded small RNAs, NA: not assessed. CSF: cerebrospinal fluid. The Kruskal-Wallis test was used for comparisons of continuous variables and the chi-square test was used for comparisons of the categorical data.

\section{Materials and Methods}

We reviewed the clinical and pathological data of consecutive cases of ARL managed at the AIDS Clinical Center, National Center for Global Health and Medicine (NCGM), Tokyo between January 1998 and June 2012. CNS involvement of systemic ARL was defined as any lesion histopathologically or radiologically diagnosed as a lymphoma in the brain, meninges, spine, cranial nerves or oculus on either initial diagnosis or recurrence. HIV-infected patients with other opportunistic infections and meningeal or parenchymal brain lesions during the same period were enrolled in the control group for the analysis. Patients who did not have available CSF samples were excluded.

Real-time polymerase chain reaction (RT-PCR) was used to quantify EBV-DNA in CSF samples obtained before chemotherapy and stored at $-80^{\circ} \mathrm{C}$, using a method previously described (11). Briefly, DNA was extracted using a QIA Symphony Virus/Bacteria Mini kit (Qiagen, Valencia, CA), and the $B N R F 1$ gene was amplified with the following primers: forward [5'-CCAGTGCTGTGATCGAGCATCT] and reverse [5'-CTGTCGACAAACTGCTGCATTC] and TaqMan probe [5'-(FAM)-TCTGCTGTGTTTCTGTCTCACCTACCG G-(TAMRA)-3']. The cutoff level for detection was 200 copies/mL.

In patients with available results of in situ hybridization (ISH) assay of EBV-encoded small RNAs (EBERs), which were performed on paraffin tissue sections using a cocktail of fluorescein-isothiocyanate-labeled oligonucleotides complementary to the two EBERs (types 1 and 2), as previously described $(12,13)$, we assessed the correlation between the results of EBER and the CNS localization of lymphoma.

Before the analysis, the levels of EBV-DNA were logtransformed and samples with undetectable EBV-DNA were considered to contain 0.0 copies $/ \mathrm{mL}$. For continuous variables, the Mann-Whitney U-test was used to compare two groups, while the Kruskal-Wallis test was applied to compare three or more groups. Categorical data were examined using the chi-square test. Differences were considered to be significant at $\mathrm{p}<0.05$. The statistical analyses were performed using the SPSS-II software package for Windows, version 17.0J (SPSS Japan Inc, Tokyo, Japan).

\section{Results}

During the study period, 76 patients were diagnosed with ARL, including eight patients with PCNSL and 68 patients with systemic ARL. One patient developed ARL twice (diffuse large B-cell lymphoma and plasmablastic lymphoma) within a several year interval and was considered to represent two systemic ARL cases. The frequency of CNS involvement in the systemic ARL patients was 22.1\% (15/68). Of the 76 patients with ARL, 62 had available CSF samples and were assigned to the analysis [PCNSL $\mathrm{n}=8$, systemic ARL with CNS involvement (ARL-CNS(+), $n=12$ ) and systemic ARL without CNS involvement (ARL-CNS(-), $n=42$ )] (Table). The 63 control subjects with definitive diagnoses of other CNS opportunistic infections during the study period consisted of 18 patients with cryptococcal meningitis, 16 patients with toxoplasmosis, 12 with progressive multifocal leukoencephalopathy (PML), five patients with cytomegalovirus (CMV) encephalitis, three patients with tuberculous meningitis, three patients with neurosyphilis, three patients with Varicella-zoster virus meningitis, two patients with HIV encephalitis, one patient with aseptic meningitis due to acute retroviral syndrome and one patient with CNS candidiasis. Three subjects in the control group had multiple opportunistic infections. There were no significant differences in sex, age or HIV viral load between the two groups. The median CD4 count in the PCNSL group was significantly lower than that observed in the group with systemic ARL with CNS involvement; however, the CD4 counts of the other groups were comparable. 


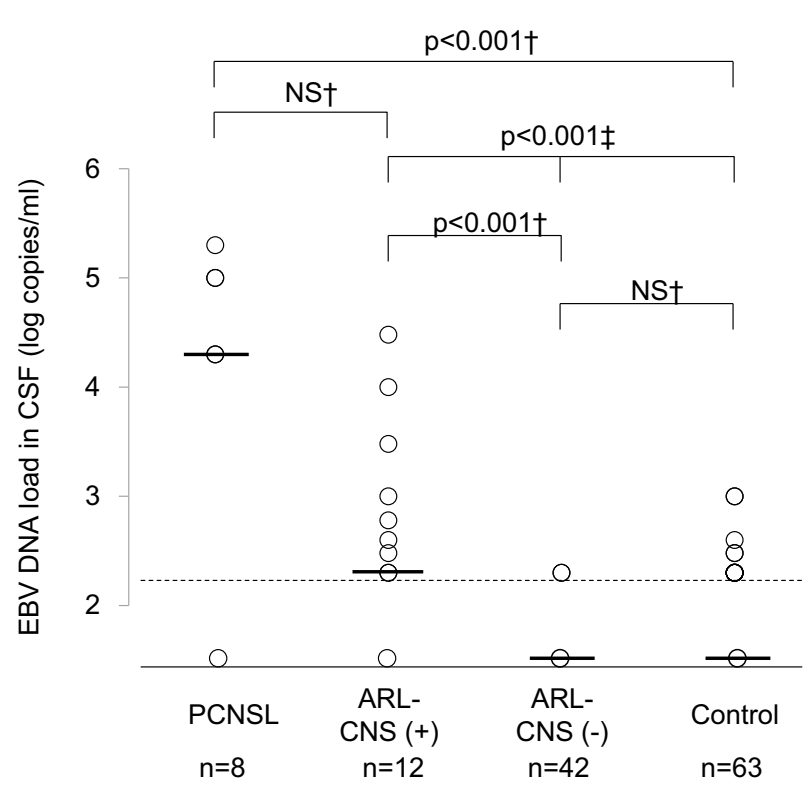

Figure 1. The EBV-DNA loads in the cerebrospinal fluid (CSF) of the patients with AIDS-related lymphoma and the control subjects. PCNSL: primary CNS lymphoma, ARL-CNS (+): systemic AIDS-related lymphoma with CNS involvement, ARL-CNS (-): systemic AIDS-related lymphoma without CNS involvement, NS: not significant. The Mann-Whitney U- test $(\dagger)$ and the Kruskal-Wallis test $(\ddagger)$ were used to compare to the EBV-DNA loads in the CSF. Individual values are plotted, and the horizontal bars represent the median values. The dotted horizontal line indicates the detection limit of the EBVDNA load assay.

The proportion of patients positive for EBV-DNA in the CSF (with a detection limit of 200 copies $/ \mathrm{mL}$ ) was $62.5 \%$ in the PCNSL, $75.0 \%$ in the ARL-CNS(+), $7.1 \%$ in the ARL-CNS(-) and $20.6 \%$ in the control group. The median (range) EBV-DNA loads in the CSF of the above groups were $4.30(0-5.30), 2.53(0-4.48), 0.00(0-2.30)$ and 0.00 (0.00-3.00) log copies $/ \mathrm{mL}$, respectively (Fig. 1). Both the rate of EBV-DNA-positive cases (Table) and the median EBV-DNA load in the CSF (Fig. 1) were significantly higher in the PCNSL and ARL-CNS(+) groups compared with those observed in the ARL-CNS(-) and control groups; however, these values were not different between the PCNSL and ARL-CNS(+) groups or between the ARLCNS(-) and control groups. Neither the detection of EBVDNA in plasma nor histological evidence of EBER in tissue were found to be correlated with the CNS localization of lymphoma (Table). Among nine EBER-negative ARLCNS(+) cases, CSF EBV-DNA was positive in the five patients who were positive for plasma EBV-DNA, while the remaining four patients were negative for both CSF and plasma EBV-DNA. Six EBER-positive ARL-CNS(+) cases included four patients with positive CSF EBV-DNA and negative plasma EBV-DNA, and one patients with positive and one patients with negative EBV-DNA in both the CSF and plasma. The concordant rate of EBV-DNA detection in the CSF and plasma was $100 \%$ in the EBER-negative in

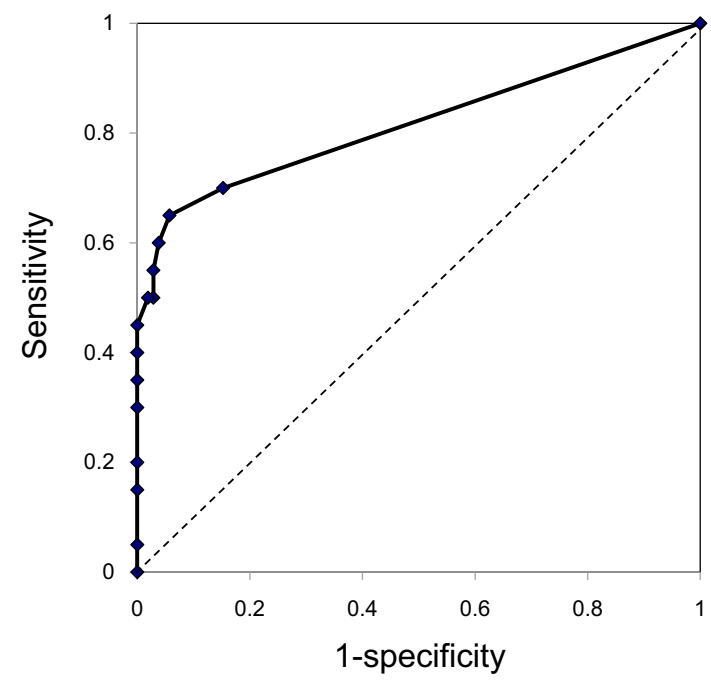

Figure 2. Receiver operating characteristic (ROC) curve for the cutoff values of the EBV-DNA load in the cerebrospinal fluid for the diagnosis of CNS involvement of systemic AIDSrelated lymphoma. The dotted line is the reference line. The area under the ROC curve was 0.856 (95\% confidence interval, 0.690-1.000). A cutoff value of $200 \mathrm{copies} / \mathrm{mL}$ had a sensitivity of $75 \%$ and a specificity of $93 \%$.

ARL-CNS(+) cases and $33 \%$ in the EBER-positive in ARL$\mathrm{CNS}(+)$ cases.

With regard to the diagnostic value of the quantitative EBV-DNA load in the CSF, a cut off value of 200 copies/ $\mathrm{mL}$ provided a sensitivity of $70 \%$ and a specificity of $85 \%$ for the CNS localization of all ARLs, including the cases of PCNSL and systemic ARL and provided a higher sensitivity of $75 \%$ and a specificity of $93 \%$ in the systemic ARL cases. A cut off value of 300 copies $/ \mathrm{mL}$ exhibited a similar sensitivity of $65 \%$ and a higher specificity of $94 \%$; however the best specificity $(100 \%)$ was noted using a cut off value of 2,000 copies $/ \mathrm{mL}$, with a sensitivity of $50 \%$. The area under the receiver operating characteristic (ROC) curve in the diagnosis of CNS localization of ARL was 0.816 for all ARLs and 0.856 for systemic ARLs (Fig. 2). Among the EBERpositive ARLs, a cut off value of 200 copies $/ \mathrm{mL}$ provid a sensitivity of $83.3 \%$ and a specificity of $90.4 \%$ in the diagnosis of CNS involvement and provide a sensitivity of $55.6 \%$ and a specificity of $100 \%$ in the EBER-negative ARL cases.

\section{Discussion}

The present study demonstrated the usefulness of measuring the EBV-DNA load in the CSF for diagnosing CNS lesions of ARL, regardless of the type of localization of lymphoma, and the presence of PCNSL or CNS involvement of systemic ARL. Although the diagnostic value of EBV-DNA for HIV-positive PCNSL is well-documented $(3-10,18)$, evidence showing its usefulness for identifying CNS lesions of systemic ARL is limited (3-10). Since the prevalence (21.7\%) of CNS involvement in patients with systemic ARL 
is considerably higher (3) than that of non-HIV lymphoma patients $(2-7 \%)(14-16)$, our results might support the clinical utility of evaluating EBV-PCR with CSF in the management of patients with HIV-positive systemic ARL.

In our study, quantitative EBV-PCR in the CSF with a cut off value of $200(2.30 \mathrm{log})$ copies/mL had a sensitivity of $70 \%$ and a specificity of $85 \%$ for the identification of lymphoma in CNS, while a cut off value of 300 copies $/ \mathrm{mL}$ provided a similar sensitivity of $65 \%$ and a higher specificity of $94 \%$. A previous study that assessed the diagnostic value of quantitative EBV-DNA assays in the CSF for identifying both systemic ARL and PCNSL (10) reported a sensitivity of $75 \%$ and specificity of $76 \%$ using a cut off value of 100 copies $/ \mathrm{mL}$, while the best specificity $(100 \%)$ was obtained using a cut off value of $3.53 \log (3,388)$ copies $/ \mathrm{mL}$. Although our study used a slightly higher detection limit and had a higher specificity and lower sensitivity, the results of the two studies are comparable. In addition, a similar sensitivity $(75 \%)$ and a higher specificity (93\%) were obtained using the cut off value of 200 copies/mL for identifying CNS involvement in systemic ARL than from among all ARLs. Overall, a cut off value of 100-300 seems to be beneficial for identifying CNS lesions of ARL.

In the present study, the prevalence of CSF EBV-DNA in the PCNSL group (62.5\%) and the EBER expression (40\%) were relatively lower than those reported previously for AIDS-related PCNSL patients (80-100\%) (3-10, 18). One possible reason for the low prevalence was the undetectable CSF EBV-DNA load in two patients who had been occasionally treated with anti-herpetic therapy before and during the treatment of PCNSL, including acyclovir for genital herpes in one patient and gancyclovir for CMV retinitis in the other (17). A history of anti-herpetic therapy should be considered when interpreting the results of EBV-PCR. In addition, most previous reports on the high rate of the EBER expression in patients with AIDS-related PCNSL were conducted before or in the early HAART era (18), enrolling severely immunocompromised patients. Since the EBER expression is rare in immunocompetent PCNSL patients (19), our results of low EBER positivity indicate changes in the characteristics of ARL among HIV patients with relatively preserved immunity in the HAART era.

In this study, we found five patients with ARL-CNS(+) who were positive for EBV-DNA in the CSF but negative for the EBER expression in tissue. Notably, among all of the patients with EBER negative ARL-CNS(+), CSF EBVDNA was detected only when plasma EBV-DNA was detectable, thus suggesting that plasma EBV-DNA transudation into CSF through the blood-brain barrier (BBB) is damaged by CNS involvement of ARL. The presence of plasma EBVDNA among ARL patients is thought to reflect EBV replication, not in lymphoma tissue, but in other lymphatic tissues such as tonsil endothelial cells, under immunosuppression (20, 21). Although increased EBV activation may lead to ARL development, the increase in the EBV-DNA load in plasma and the EBER expression in tissue are not fully syn- chronized $(20,21)$. This may explain our finding of EBERnegative but CSF EBV-DNA positive ARL. Since our study is retrospective, the residue of specimens for EBER ISH was unavailable in $25 \%$ of the patients with CNS involvement. Further studies are needed to understand the role of CSF EBV-DNA measurement in the context of EBER-negative ARL.

\section{Conclusion}

The EBV-DNA load in the CSF is a marker of CNS involvement of ARL, with 200 copies $/ \mathrm{mL}$ being a cut off level for the diagnosis of PCNSL and the identification of CNS involvement in patients with systemic ARL. Identifying EBV-DNA may help to differentiate the CNS lesions of ARL from other disorders.

The authors state that they have no Conflict of Interest (COI).

\section{Acknowledgement}

We thank the entire staff of the AIDS Clinical Center for caring for the patients.

This work was supported by a Grant for International Health Research (A21-104) and the Health Labour Sciences Research Grant (H22-AIDS-002) from the Ministry of Health, Labour and Welfare.

\section{References}

1. Vaccher E, Spina M, Talamini R, et al. Improvement of systemic human immunodeficiency virus-related non-Hodgkin's lymphoma outcome in the era of highly active antiretroviral therapy. Clin Infect Dis 37: 1556-1564, 2003.

2. Desai J, Mitnick RJ, Henry DH, et al. Patterns of central nervous system recurrence in patients with systemic human immunodeficiency virus-associated non-Hodgkin lymphoma. Cancer 86: 18401847, 1999.

3. Cingolani A, Gastaldi R, Fassone L, et al. Epstein-Barr virus infection is predictive of CNS involvement in systemic AIDS-related non-Hodgkin's lymphomas. J Clin Oncol 18: 3325-3330, 2000.

4. Cinque P, Brytting M, Vago L, et al. Epstein-Barr virus DNA in cerebrospinal fluid from patients with AIDS-related primary lymphoma of the central nervous system. Lancet 342: 398-401, 1993.

5. Arribas JR, Clifford DB, Fichtenbaum CJ, et al. Detection of Epstein-Barr virus DNA in cerebrospinal fluid for diagnosis of AIDS-related central nervous system lymphoma. J Clin Microbiol 33: 1580-1583, 1995.

6. Roberts TC, Storch GA. Multiplex PCR for diagnosis of AIDSrelated central nervous system lymphoma and toxoplasmosis. J Clin Microbiol 35: 268-269, 1997.

7. Antinori A, De Rossi G, Ammassari A, et al. Value of combined approach with thallium-201 single-photon emission computed tomography and Epstein-Barr virus DNA polymerase. J Clin Oncol 17: 554-560, 1999.

8. Ivers LC, Kim AY, Sax PE. Predictive value of polymerase chain reaction of cerebrospinal fluid for detection of Epstein-Barr virus to establish the diagnosis of HIV-related primary central nervous system lymphoma. Clin Infect Dis 38: 1629-1632, 2004.

9. Tachikawa N, Goto M, Hoshino Y, et al. Detection of Toxoplasma gondii, Epstein-Barr virus, and JC virus DNAs in the cerebrospinal fluid in acquired immunodeficiency syndrome patients with 
focal central nervous system complications. Intern Med 38: 556$562,1999$.

10. Bossolasco S, Cinque P, Ponzoni $M$, et al. Epstein-Barr virus DNA load in cerebrospinal fluid and plasma of patients with AIDS-related lymphoma. J Neurovirol 8: 432-438, 2002.

11. Shide K, Henzan H, Nagafuji $K$, et al. Dynamics of Epstein-Barr virus load in pyothorax-associated lymphoma. J Med Virol 70: 137-140, 2003.

12. Gulley ML, Glaser SL, Craig FE, et al. Guidelines for interpreting EBER in situ hybridization and LMP1 immunohistochemical tests for detecting Epstein-Barr virus in Hodgkin lymphoma. Am J Clin Pathol 117: 259-267, 2002.

13. Khan G, Coates PJ, Kangro HO, et al. Epstein Barr virus (EBV) encoded small RNAs: targets for detection by in situ hybridisation with oligonucleotide probes. J Clin Pathol 45: 616-620, 1992.

14. Boehme V, Zeynalova $S$, Kloess $M$, et al. Incidence and risk factors of central nervous system recurrence in aggressive lymphoma - a survey of 1693 patients treated in protocols of the German High-Grade Non-Hodgkin's Lymphoma Study Group (DSHNHL). Ann Oncol 18: 149-157, 2007.

15. Bjorkholm M, Hagberg $H$, Holte $H$, et al. Central nervous system occurrence in elderly patients with aggressive lymphoma and a long-term follow-up. Ann Oncol 18: 1085-1089, 2007.

16. Besien KV, Ha CS, Murphy S, et al. Risk factors, treatment, and outcome of central nervous system recurrence in adults with intermediate-grade and immunoblastic lymphoma. Blood 91: 1178-1184, 1998.

17. Bossolasco S, Falk KI, Ponzoni M, et al. Ganciclovir is associated with low or undetectable Epstein-Barr virus DNA load in cerebrospinal fluid of patients with HIV-related primary central nervous system lymphoma. Clin Infect Dis 42: e21-e25, 2006.

18. MacMahon EM, Glass JD, Hayward SD, et al. Epstein-Barr virus in AIDS-related primary central nervous system lymphoma. Lancet 338: 969-973, 1991.

19. Wada N, Ikeda J, Hori Y, et al. Epstein-Barr virus in diffuse large B-Cell lymphoma in immunocompetent patients in Japan is as low as in Western Countries. J Med Virol 83: 317-321, 2011.

20. Van Baarle D, Wolthers KC, Hovenkamp E, et al. Absolute level of Epstein-Barr virus DNA in human immunodeficiency virus type 1 infection is not predictive of AIDS-related non-Hodgkin lymphoma. J Infect Dis 186: 405-409, 2002.

21. Gan YJ, Razzouk BI, Su T, Sixbey JW. A defective, rearranged Epstein-Barr virus genome in EBER-negative and EBER-positive Hodgkin's disease. Am J Pathol 160: 781-786, 2002.

(C) 2013 The Japanese Society of Internal Medicine http://www.naika.or.jp/imonline/index.html 